\title{
Sensibilidade de cultivares de algodoeiro ao cloreto de mepiquat
}

\author{
Julio Cesar Bogiani(1) e Ciro Antonio Rosolem ${ }^{(1)}$
}

(1)Universidade Estadual Paulista, Faculdade de Ciências Agronômicas, Campus de Botucatu, Caixa Postal 237, CEP $18603-970$ Botucatu, SP.

E-mail: jbogiani@fca.unesp.br, rosolem@fca.unesp.br

\begin{abstract}
Resumo - $\mathrm{O}$ objetivo deste trabalho foi avaliar a sensibilidade de cultivares de algodoeiro ao cloreto de mepiquat e verificar se ela está relacionada ao ciclo ou à arquitetura da planta. Foi realizado um experimento em casa de vegetação com seis cultivares de algodoeiro - FiberMax 966, FiberMax 977, Delta Penta, Delta Opal, FMT 501 e FMT 701 - e quatro doses do regulador vegetal à base de cloreto de mepiquat $-0,7,5,15$ e $22,5 \mathrm{~g}^{-1}{ }^{-1}$ de i.a. -, em delineamento de blocos ao acaso, com arranjo fatorial 6x4 e quatro repetições. As parcelas experimentais foram constituídas por vasos de $12 \mathrm{~L}$ com duas plantas em cada um. As doses do regulador vegetal foram aplicadas sem adjuvante, no estádio de aparecimento dos botões florais, aos 34 dias após a emergência das plântulas. Paralelamente ao experimento com doses, a retenção do regulador pelas folhas das cultivares foi quantificada. O crescimento em altura das plantas de algodoeiro é diminuído com a aplicação do regulador vegetal, e esse efeito se intensifica com o aumento da dosagem aplicada. Existe diferença entre as cultivares estudadas quanto à sensibilidade ao cloreto de mepiquat, sendo que as mais precoces são mais sensíveis. Além da sensibilidade diferenciada, a arquitetura das plantas pode possibilitar maior ou menor deposição do regulador sobre as folhas.
\end{abstract}

Termos para indexação: Gossypium hirsutum, altura de planta, crescimento de planta, regulador vegetal.

\section{Sensibility of cotton cultivars to mepiquat chloride}

\begin{abstract}
The objective of this work was to assess the sensibility of cotton cultivars to mepiquat chloride and to verify if it is related to plant cycle or architecture. A green-house experiment was carried out with six cotton cultivars - FiberMax 966, FiberMax 977, Delta Penta, Delta Opal, FMT 501 e FMT 701 -, and four concentrations of mepiquat chloride plant growth regulator $-0,7,5,15$ and 22,5 $\mathrm{g} \mathrm{ha}^{-1}$ of the active ingredient - in complete block design with a $6 \times 4$ factorial arrangement and four replicates. The plots consisted of $12 \mathrm{~L}$ pots with two plants per pot. Regulator doses were applied without adjuvants at the beginning of flowering, 34 days after emergence of seedlings. Besides the experiment with doses, the regulator deposition on leaves of each cultivar was quantified. The growth regulator reduces the growth of cotton plants, and this effect is intensified by increasing the dosage. There are differences among cotton cultivars regarding their sensibility to mepiquat chloride, with the earliest cultivars being the most sensitive. Besides differential sensibility, cultivar architecture can provide greater or lesser regulator deposition on leaves.
\end{abstract}

Index terms: Gossypium hirsutum, plant height, plant growth, plant growth regulator.

\section{Introdução}

O algodoeiro é uma espécie originalmente perene, de crescimento indeterminado e contínuo, que emite sucessivas gemas terminais, a não ser que seja interrompido por fatores externos (Beltrão \& Souza, 2001). Atualmente, no entanto, é cultivado como cultura anual na maioria dos países produtores.

A cotonicultura brasileira passou por mudanças expressivas quando comparada à da década de 1980 . A tecnologia preconizada atualmente tem entre as principais recomendações a adequada correção do solo e a utilização de fertilizantes (Carvalho et al., 2007).
Nessas condições, normalmente ocorre crescimento excessivo das plantas, que atingem alturas indesejáveis para a colheita mecanizada, e produção de grande volume de folhas, o que pode resultar em diminuição da produtividade (Cook \& Kennedy, 2000; Nichols et al., 2003). A manipulação da arquitetura das plantas do algodoeiro com uso de reguladores vegetais é uma das tecnologias recomendadas para evitar essa queda de produtividade (Azevedo et al., 2004).

Os reguladores vegetais são substâncias químicas sintéticas que alteram o balanço hormonal das plantas. $\mathrm{O}$ cloreto de mepiquat é absorvido principalmente pelas partes verdes da planta e pode ser incluído no 
grupo de inibidores da biossíntese do ácido giberélico, o que o torna, portanto, um inibidor do alongamento celular (Lamas, 2001). No algodoeiro, sua aplicação visa reduzir a altura da planta, para torná-la mais compacta e facilitar o uso de defensivos e a colheita mecânica (Marur, 1998; Lamas et al., 2000). Os principais efeitos do cloreto de mepiquat sobre o algodoeiro são: redução no crescimento, no número e no tamanho dos ramos reprodutivos, e melhoria da arquitetura e das proporções entre as partes vegetativas e reprodutivas (Reddy et al., 1990; Athayde \& Lamas, 1999; Soares, 1999; Stewart et al., 2001).

A definição da dose adequada a ser aplicada é uma das principais dificuldades na recomendação de reguladores de crescimento (Athayde \& Lamas, 1999), uma vez que nem sempre são atingidos os resultados esperados com o uso das doses recomendadas. A diversidade de cultivares atualmente em uso no Brasil faz com que a previsibilidade do resultado da aplicação desses produtos seja reduzida (Zanon, 2002), já que podem existir, entre elas, diferenças de sensibilidade ao regulador aplicado, conforme observaram Gwathmey \& Craig Junior (2003). Existem, atualmente, cultivares de algodoeiro de ciclo precoce, médio e tardio, com diferentes arquiteturas e conformações, que demandam diferentes manejos do regulador vegetal (Ornellas et al., 2001). É possível que as diferenças de sensibilidade ao regulador estejam relacionadas ao porte da planta (alto, médio e baixo) e ao ciclo do material recomendado (precoce, médio e tardio).

O presente trabalho teve como objetivo investigar a sensibilidade de cultivares de algodoeiro, com diferentes características de arquitetura e ciclo produtivo, ao uso de doses de cloreto de mepiquat, bem como verificar se essa sensibilidade está ligada ao ciclo ou à arquitetura da cultivar.

\section{Material e Métodos}

O experimento foi conduzido em casa de vegetação no Departamento de Produção Vegetal da Faculdade de Ciências Agronômicas da Universidade Estadual Paulista, Botucatu, SP.

O solo utilizado foi classificado como Latossolo Vermelho distroférrico, de textura média: $630 \mathrm{~g} \mathrm{~kg}^{-1} \mathrm{de}$ areia, $40 \mathrm{~g} \mathrm{~kg}^{-1}$ de silte e $330 \mathrm{~g} \mathrm{~kg}^{-1}$ de argila (Sistema brasileiro de classificação de solos, 1999). A análise química (Raij et al., 2001) revelou os seguintes valores:
$\mathrm{pH}\left(\mathrm{CaCl}_{2}\right) 4,3 ; 0,1 \mathrm{mg} \mathrm{dm}^{-3}$ de P (resina); $15,9 \mathrm{~g} \mathrm{dm}^{-3}$ de matéria orgânica; 70,4 $\mathrm{mmol}_{\mathrm{c}} \mathrm{dm}^{-3}$ de $\mathrm{H}+\mathrm{Al}$; $1 \mathrm{mmol}_{\mathrm{c}} \mathrm{dm}^{-3} \mathrm{deK} ; 3 \mathrm{mmol}_{\mathrm{c}} \mathrm{dm}^{-3} \mathrm{de} \mathrm{Ca} ; 0,5 \mathrm{mmol}_{\mathrm{c}} \mathrm{dm}^{-3} \mathrm{de}$ $\mathrm{Mg} ; 4,5 \mathrm{mmol}_{\mathrm{c}} \mathrm{dm}^{-3} \mathrm{de}$ soma de bases; 74,9 $\mathrm{mmol}_{\mathrm{c}} \mathrm{dm}^{-3}$ de CTC; e $6 \%$ de saturação por bases. Foi aplicado calcário dolomítico $(\mathrm{CaO}, 28 \%$; $\mathrm{MgO}, 20 \%$ e PRNT, $91 \%$ ), para elevar a saturação por bases a $60 \%$. O solo previamente seco ao ar e peneirado em peneira com malha de $4 \mathrm{~mm}$ foi umedecido até a capacidade de campo e acondicionado sob lona plástica por 20 dias. Em seguida, foi adubado com $150 \mathrm{mg} \mathrm{dm}^{-3}$ de $\mathrm{P}_{2} \mathrm{O}_{5}$ (superfosfato simples), $150 \mathrm{mg} \mathrm{dm}^{-3} \mathrm{de}_{2} \mathrm{O}$ (cloreto de potássio), $50 \mathrm{mg} \mathrm{dm}^{-3}$ de nitrogênio (ureia), $2 \mathrm{mg} \mathrm{dm}^{-3}$ de zinco (sulfato de zinco) e $2 \mathrm{mg} \mathrm{dm}^{-3}$ de boro (ácido bórico).

As sementes foram submersas em água destilada por 36 horas, para pré-germinação. Foram utilizadas seis sementes em cada vaso. A emergência ocorreu quatro dias após a semeadura e, passados outros cinco dias, foi realizado desbaste para duas plantas por vaso. Os vasos tinham capacidade para $12 \mathrm{~L}$. O teor de água no solo foi corrigido diariamente, com base em pesagens de vasos controle. Por ocasião do desbaste, foi realizada a primeira aplicação de solução nutritiva (Hoagland \& Arnon, 1950) diluída em $50 \%$ e, a partir de então, foi aplicada semanalmente a solução completa, até o final do experimento. $\mathrm{O}$ uso de solução nutritiva é necessário para evitar, pelo menos parcialmente, o efeito de vaso no crescimento das plantas. Essa prática foi utilizada com base nos resultados de Souza \& Rosolem (2007).

Os tratos culturais constaram do controle de plantas daninhas e da aplicação de inseticida para o controle de pulgão (Aphis sp.), tripes (Frankliniella sp.) e mosca-branca (Bemisia sp.) aos 25 e 48 DAE.

Os tratamentos foram constituídos por seis cultivares de algodoeiro e quatro doses de regulador vegetal à base de cloreto de mepiquat (produto comercial com $50 \mathrm{~g} \mathrm{~L}^{-1}$ de i.a.). As cultivares utilizadas foram: Fibermax 966 (FMX 966 - ciclo precoce/médio e porte médio/baixo); Fibermax 977 (FMX 977 - ciclo médio/tardio e porte médio/alto); FMT 501 (ciclo precoce/médio e porte médio/ alto); Delta Penta (ciclo médio/tardio e porte alto); Delta Opal (ciclo tardio e porte alto) e FMT 701 (ciclo tardio e porte alto). As doses do regulador corresponderam a 0 , $7,5,15$ e 22,5 $\mathrm{g} \mathrm{ha}^{-1}$ do ingrediente ativo, aplicadas sem adjuvante no estádio de aparecimento dos botões florais (Biles \& Cothren, 2001), aos 34 dias após a emergência das plântulas (DAE). O delineamento experimental foi o

Pesq. agropec. bras., Brasília, v.44, n.10, p.1246-1253, out. 2009 
de blocos ao acaso, em arranjo fatorial com quatro doses e seis cultivares, com quatro repetições.

Para pulverização dos tratamentos, foram utilizadas pontas do tipo cônico vazio (JA-2, Jacto, São Paulo), pressão de $60 \mathrm{lbf} \mathrm{pol}^{-2}$ e volume de $150 \mathrm{~L} \mathrm{ha}^{-1}$ de calda. Antes da aplicação do regulador vegetal, mediu-se a altura de todas as plantas (do colo até o último nó vegetativo). A organização das unidades experimentais ao longo do percurso da barra de pulverização foi feita utilizando espaçamento de $0,90 \mathrm{~m}$, simulando uma população de 90 mil plantas por hectare.

Após a aplicação dos tratamentos às plantas, foram realizadas medições de altura durante 30 dias, em intervalos de três dias, até que as plantas fossem colhidas, aos 64 DAE. Na colheita do experimento, foi avaliado o número de ramos reprodutivos e feita a contagem do número de internódios. Pela razão entre a altura final das plantas e o número de internódios, obteve-se o comprimento médio de internódios.

Durante o período de condução do experimento, foram registradas as temperaturas máxima $\left(\mathrm{T}_{\text {máx }}\right)$ e mínima $\left(\mathrm{T}_{\text {min }}\right)$ do ar dentro da casa de vegetação, por meio de um termo-higrógrafo, para o cálculo dos graus-dia acumulados (GD). Com as médias de altura das plantas (coletadas a cada três dias), determinou-se o crescimento de acordo com os graus-dia acumulados. No cálculo de GD, foi considerada a temperatura basal mínima (TB) de $15^{\circ} \mathrm{C}$, utilizando-se a equação GD $=\left(\mathrm{T}_{\text {máx }}+\mathrm{T}_{\text {min }}\right) / 2-\mathrm{TB}$ (Oosterhuis, 1992).

Paralelamente ao experimento com doses e cultivares, foi efetuado um estudo de retenção do regulador pelas folhas do algodoeiro com uso da metodologia descrita por Palladini (2000). Empregou-se o delineamento em blocos ao acaso, com seis cultivares e quatro repetições. Nos tratamentos com doses e cultivares, foi aplicada às plantas do estudo paralelo a calda com corante azul FDC-1 (azul brilhante), na concentração de $3.000 \mathrm{mg} \mathrm{L}^{-1}$. As folhas provenientes dessas plantas foram coletadas e lavadas em $300 \mathrm{~mL}$ de água destilada. Foi medida a massa de matéria seca das plantas, bem como a área foliar e a altura. A água proveniente da lavagem das folhas foi coletada e submetida à leitura de absorbância em espectrofotômetro, para se estimar a quantidade de produto presente na água de lavagem e, por consequência, a quantidade retida nas folhas. Pela relação entre a quantidade de produto retirada por unidade de área foliar, a área foliar total das plantas e a massa de matéria seca por ocasião da aplicação do produto, foi estimada a concentração do produto na planta, assumindo-se que o total aplicado tenha sido absorvido.

Os dados foram submetidos à análise de variância. As médias dos tratamentos foram comparadas pelo teste t, a $5 \%$ de probabilidade, e foi feita análise de regressão para as doses. No estudo de crescimento das cultivares dentro de cada dose, foi usada análise de regressão, e considerou-se como fator quantitativo os graus-dia acumulados. As taxas de crescimento foram calculadas como sendo a primeira derivada das equações ajustadas ao crescimento acumulado das plantas de algodão. Por meio da análise de regressão entre a taxa de crescimento e a concentração de regulador vegetal na planta, foi determinada a sensibilidade de cada cultivar ao regulador.

\section{Resultados e Discussão}

Todas as cultivares tiveram a altura final diminuída com a aplicação de cloreto de mepiquat, e esse efeito se intensificou com o aumento da quantidade de regulador aplicada (Figura 1). Em geral, a cultivar FMX 966 apresentou menor altura final que as demais. Deve-se salientar, no entanto, que ela é uma cultivar de porte baixo e que, mesmo no tratamento sem aplicação do regulador, já apresentava altura significativamente menor que as cultivares Delta Opal, FMT 501 e Delta Penta.

As cultivares Delta Penta e FMT 501, que no tratamento controle apresentaram altos valores de altura final, sofreram redução mais acentuada nesses valores com uso de regulador. Delta Opal é uma cultivar de porte alto e apresentou os maiores valores, tanto no tratamento sem aplicação de regulador quanto em todas as doses de cloreto de mepiquat utilizadas (Figura 1). No tratamento controle, ela diferiu significativamente apenas de 'FMX 966', mas, com a maior dose, diferiu também de outras três cultivares: FMT 701, Delta Penta e FMT 501. De acordo com York (1983), o cloreto de mepiquat reduz a concentração de ácido giberélico das plantas de algodão por meio da inibição de sua síntese. Como esse hormônio é responsável pela divisão e expansão celular (Taiz \& Zeiger, 2004), o bloqueio ou diminuição de sua síntese causa redução na altura das plantas.

Foram observadas diferenças entre as cultivares também no número de internódios, sendo que a cultivar FMX 966 apresentou menor número de internódios que a Delta Opal, em todas as doses de regulador utilizadas, 
pela comparação com o teste t, a $5 \%$ de probabilidade. As cultivares FMT 501 e 701 foram as mais afetadas pela utilização do regulador vegetal, pois apresentaram maiores inclinações na regressão linear (Figura 1). A diminuição no número de internódios pela aplicação de cloreto de mepiquat tem sido comumente relatada na cultura do algodoeiro (Reddy et al., 1996; Soares, 1999; Souza, 2004).

Observou-se que o uso de doses de cloreto de mepiquat reduziu linearmente também o comprimento dos internódios de todas as cultivares (Figura 1). A cultivar FMX 966 apresentou o menor valor na dose 22,5 $\mathrm{g} \mathrm{ha}^{-1}$ de i.a., seguida das cultivares Delta Penta, FMT 701, FMX 977, FMT 511 e Delta Opal. Dessas, as únicas que não diferiram estatisticamente da 'Delta Opal' foram as cultivares FMT 501 e FMX 977. McCarty Junior \& Hedin (1994) também observaram que a aplicação de cloreto de mepiquat no algodoeiro reduziu a altura das plantas por meio do encurtamento dos internódios, o que resultou em plantas mais compactas, com as maçãs localizadas nos ramos mais baixos, e em encurtamento do ciclo.

O número de ramos reprodutivos foi diminuído com o aumento da dose de regulador vegetal (Figura 1), exceto para as cultivares FMX 966 e 977, que não apresentaram variação significativa para o efeito das doses pelo teste t. Foram observadas diferenças entre as cultivares no tratamento sem regulador, e a FMX 966 apresentou menor quantidade de ramos reprodutivos que as demais, sem diferir significativamente de 'Delta Penta'. Já na maior dose, essas duas cultivares apresentaram quantidades significativamente menores de ramos reprodutivos que a 'FMT 977'.

Observa-se que, de maneira geral, o crescimento do algodoeiro após a aplicação foi maior no tratamento sem aplicação do regulador, e foi reduzido com as doses de regulador vegetal (Figura 2). A aplicação da dose de $22,5 \mathrm{~g} \mathrm{ha}^{-1}$ de i.a. foi a que proporcionou maiores diferenças de crescimento entre as cultivares. Na dose de $0 \mathrm{~g} \mathrm{ha}^{-1}$ de i.a., observou-se maior crescimento da
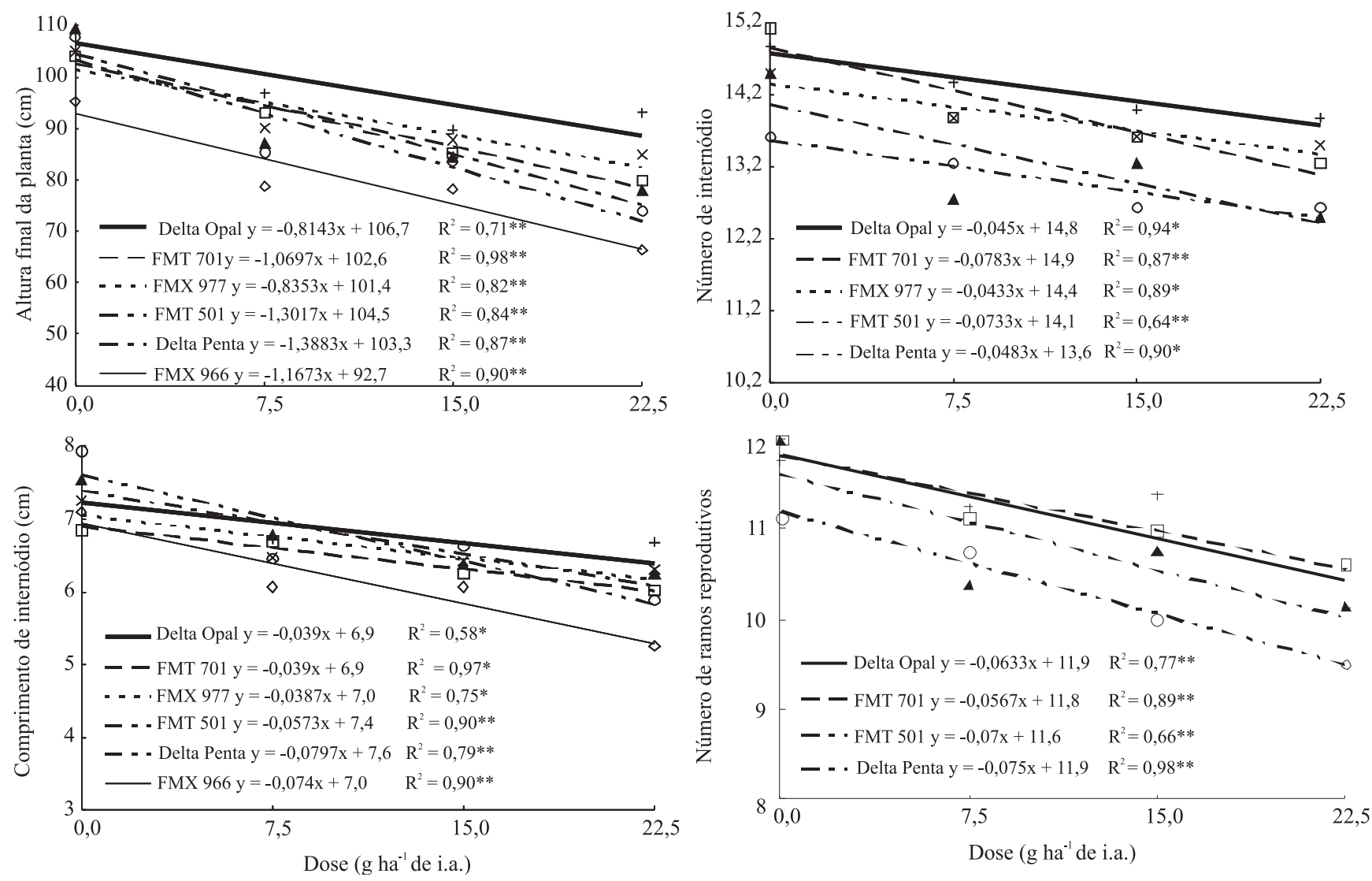

Figura 1. Altura (DMS 5\%, $10 \mathrm{~cm}$ ), número de internódios (DMS 5\%,1), comprimento médio de internódios (DMS 5\%, 0,7 cm) e número de ramos reprodutivos por planta de algodoeiro (DMS 5\%, 1) das cultivares Delta Opal (+), FMT 701 ( $\square$ ), FMX 977 $(\mathrm{x})$, FMT 501( $\Delta$ ), Delta Penta (०), FMX $966(\diamond)$, em função das doses de cloreto de mepiquat. ** e *Significativo a 1 e $5 \%$ de probabilidade, respectivamente, para o modelo linear. 
cultivar Delta Opal, menor crescimento de 'FMX 966' e, também, que o crescimento das cultivares FMX 977, FMT 501 e 701 foi semelhante (Figura 2 A). Na dose de $7,5 \mathrm{~g} \mathrm{ha}^{-1}$ de i.a., a cultivar Delta Opal também mostrou o maior crescimento, seguida das cultivares FMT 701, FMX 977 e FMT 501 (Figura 2 B). O crescimento das cultivares FMX 966 e Delta Penta foi muito parecido e apresentou os menores valores nessa dose. Na dose de $15 \mathrm{~g} \mathrm{ha}^{-1}$ de i.a., o crescimento das cultivares foi bastante semelhante, destacando-se somente a cultivar Delta Penta, que apresentou menor crescimento em comparação com as demais (Figura $2 \mathrm{C}$ ). Para a dose de 22,5 $\mathrm{g} \mathrm{ha}^{-1}$ de i.a., as plantas cresceram menos em relação às doses anteriores e houve mais diferenças entre as cultivares (Figura 2 D). Isso era esperado, já que a redução do crescimento das plantas é proporcional à dose aplicada (Lamas, 2001; Azevedo et al., 2004) e a resposta de redução é variável entre as cultivares (York, 1983; Cathey \& Meredith Junior, 1988; Gwathmey \& Craig Junior, 2003). Ainda nessa dose, pôde-se observar claramente maior crescimento da cultivar Delta Opal. As cultivares Delta Penta e FMT 501 foram equivalentes quanto ao crescimento e, na dose de 22,5 $\mathrm{g} \mathrm{ha}^{-1}$, ambas apresentaram grande redução na taxa de crescimento. A cultivar que exibiu o menor crescimento após a maior dose de cloreto de mepiquat foi FMX 966 (Figura 2 D). Esses resultados estão de acordo com os obtidos por York (1983), que também constatou que o regulador vegetal reduz o crescimento das plantas de algodoeiro e que as cultivares respondem de forma diferenciada à aplicação do regulador.
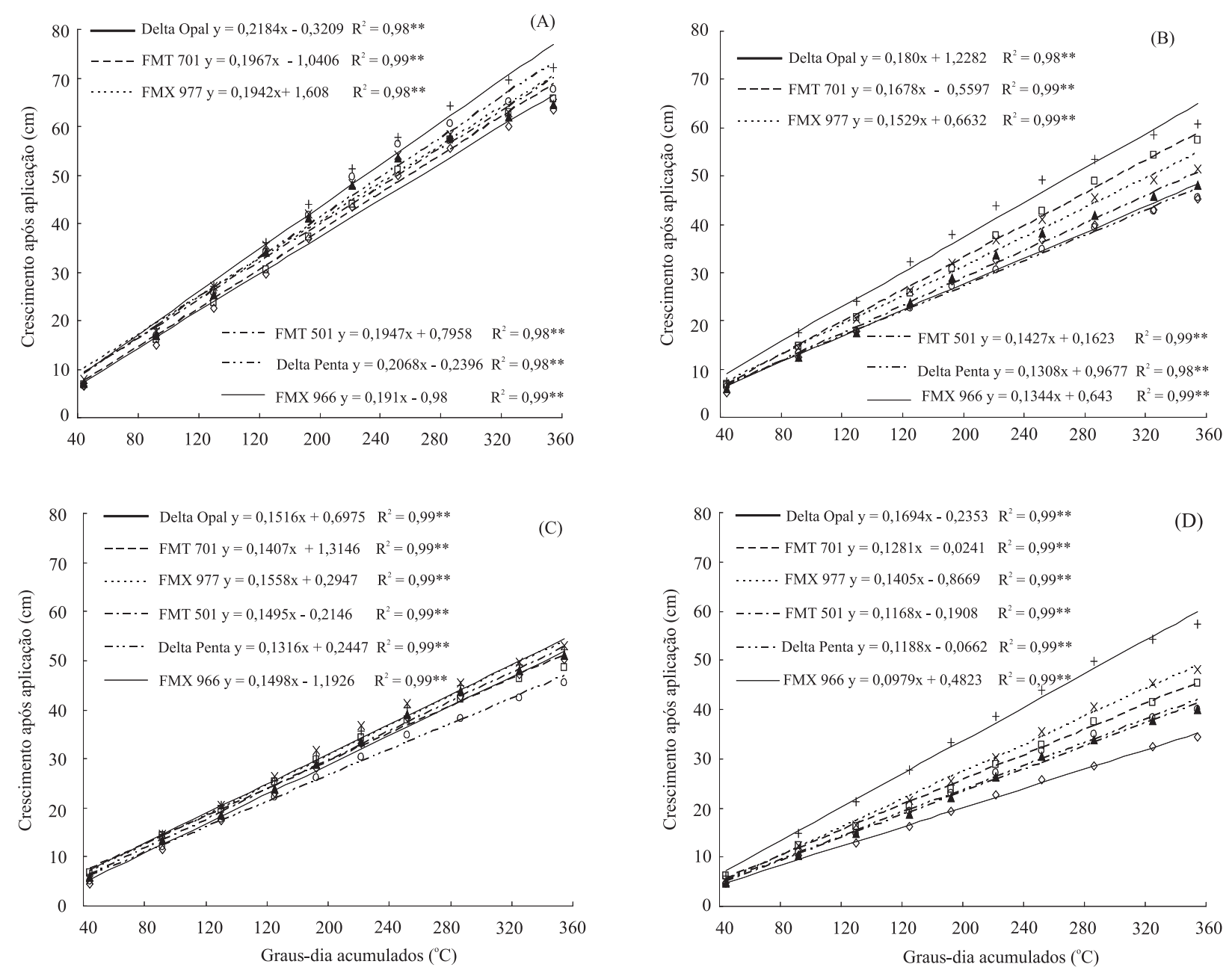

Figura 2. Crescimento das cultivares de algodoeiro Delta Opal (+), FMT $701(\square)$, FMX 977 (x), FMT 501( $\mathbf{\Delta})$, Delta Penta (○), FMX $966(\diamond)$ após aplicação de cloreto de mepiquat nas doses 0 (A), 7,5 (B), 15 (C) e 22,5 g ha-1 de i.a. (D), em função dos graus-dia acumulados. **Significativo a $1 \%$ de probabilidade, para o modelo linear. 
Com relação à concentração de regulador vegetal no tecido das plantas de algodoeiro, nota-se que houve incremento linear com o aumento da dose aplicada em todas as cultivares (Figura 3). Na dose de 7,5 $\mathrm{g} \mathrm{ha}^{-1} \mathrm{de}$ i.a., não houve diferença entre as cultivares quanto à concentração do cloreto de mepiquat pelo teste $t$. Já na dose de $15 \mathrm{~g} \mathrm{ha}^{-1}$ de i.a., a cultivar FMT 701 apresentou concentração de regulador maior do que as demais, com exceção da FMX 966. Na dose de 22,5 g ha $^{-1}$ de i.a., a concentração observada para a cultivar FMT 701 foi a maior em comparação às demais cultivares, ou seja, sua arquitetura possibilitou maior deposição do produto, o que pode ser bem visualizado na Figura 3. Ainda na maior dose, a cultivar Delta Penta apresentou a menor concentração de cloreto de mepiquat .

Houve redução linear da taxa de crescimento das plantas com o aumento da concentração do produto, e a sensibilidade das cultivares à ação do regulador diferiu claramente (Figura 4), o que corrobora os resultados obtidos por Reddy et al. (1996). Souza (2004) também notou redução linear da taxa de crescimento com o aumento da concentração do regulador no tecido da planta de algodoeiro e demonstrou que, para uma redução a ordem de $0,1 \mathrm{~cm} \mathrm{GD}^{-1}$, foi necessária uma concentração de $17,59 \mu \mathrm{g} \mathrm{g}^{-1}$ de cloreto de mepiquat.

A sensibilidade das cultivares ao cloreto de mepiquat é representada pelo coeficiente angular

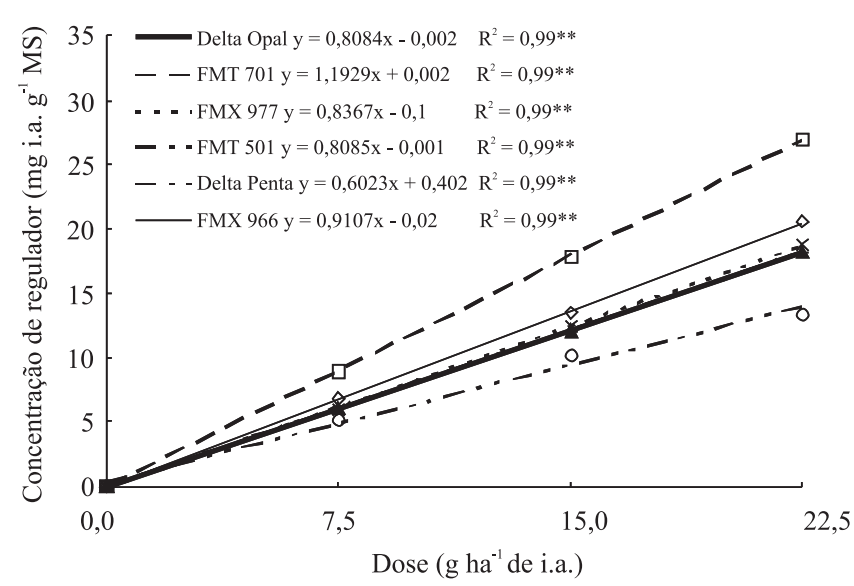

Figura 3. Concentração de regulador vegetal no tecido de plantas de algodoeiro (DMS 5\%, 4,8 $\mu$ g de i.a. $\mathrm{g}^{-1}$ de matéria seca) das cultivares Delta Opal (+), FMT $701(\square)$, FMX

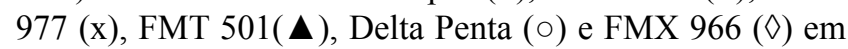
função das doses de cloreto de mepiquat. ${ }^{*}$ Significativo a $1 \%$ de probabilidade, para o modelo linear. das retas exibidas na Figura 4, ou seja, quanto maior o coeficiente angular, maior a sensibilidade. Observou-se que, apesar de a cultivar FMT 701 ter apresentado uma grande concentração do produto, sua sensibilidade ao cloreto de mepiquat equiparou-se à da 'FMX 977', e os coeficientes angulares nessas cultivares foram os menores observados $(-0,0026$ e $-0,0025$, respectivamente), o que indica baixa sensibilidade ao regulador. A cultivar Delta Penta apresentou a maior sensibilidade, com um coeficiente angular de -0,0051. É possível observar, na Figura 4, que as cultivares de ciclo mais curto (FMX 966, FMT 501 e Delta Penta) apresentaram coeficientes angulares com valores inferiores aos das cultivares de ciclo mais tardio (FMX 977, Delta Opal e FMT 701). É possível, portanto, inferir que a sensibilidade existente entre os materiais tem relação com o ciclo, ou seja, que as cultivares mais precoces apresentam maior sensibilidade ao cloreto de mepiquat do que as mais tardias. No entanto, a quantidade de produto depositado nas folhas de cada cultivar não apresentou o mesmo comportamento (Figura 3), com as maiores concentrações sendo observadas na 'FMT 701' e na 'FMX 966', que são tardia e precoce, respectivamente. $\mathrm{Na}$ dose mais alta, a cultivar FMT 701 diferiu significativamente de todas as demais, e a 'FMX 966' diferiu apenas da 'Delta Penta'.

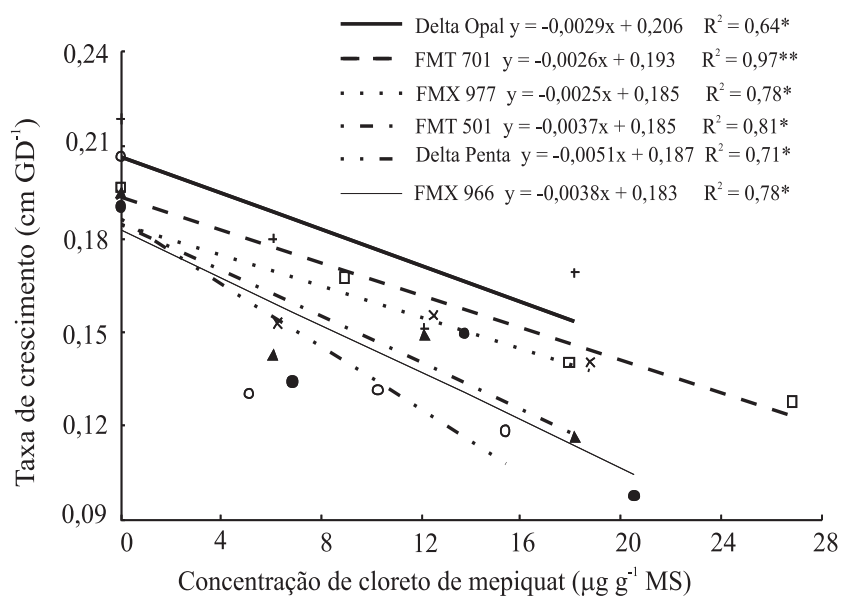

Figura 4. Sensibilidade de cultivares de algodoeiro Delta Opal (+), FMT 701 (), FMX 977 (x), FMT 501( ( ), Delta Penta (०) e FMX $966(\bullet)$, expressa pela relação entre a taxa de crescimento e a concentração de cloreto de mepiquat na massa de matéria seca de folhas. ** e *Significativo a 1 e $5 \%$ de probabilidade, para o modelo linear.

Pesq. agropec. bras., Brasília, v.44, n.10, p.1246-1253, out. 2009 


\section{Conclusões}

1. O crescimento do algodoeiro em altura é diminuído com a aplicação foliar de cloreto de mepiquat, e essa redução é proporcional à dose aplicada.

2. A sensibilidade ao cloreto de mepiquat é maior em cultivares mais precoces.

3. O crescimento do algodoeiro em resposta à aplicação do cloreto de mepiquat depende da sensibilidade das cultivares a esse regulador vegetal $\mathrm{e}$ da quantidade de produto que a arquitetura das plantas permite que seja depositada nas folhas.

\section{Referências}

ATHAYDE, M.L.F.; LAMAS, F.M. Aplicação seqüencial de cloreto de mepiquat em algodoeiro. Pesquisa Agropecuária Brasileira, v.34, p.369-375, 1999.

AZEVEDO, D.M.P. de; BEZERRA, J.R.C.; SANTOS, J.W. dos; DIAS, J.M.; BRANDÃO, Z.N. Efeito do parcelamento do cloreto de mepiquat em algodoeiro irrigado no Nordeste brasileiro. Revista Brasileira de Oleaginosas e Fibrosas, v.8, p.823-830, 2004.

BELTRÃO, N.E.M.; SOUZA, J.G. Fisiologia e ecofisiologia do algodoeiro. In: ALGODÃO: tecnologia de produção. Dourados: Embrapa Agropecuária Oeste; Campina Grande: Embrapa Algodão, 2001. p.54-75.

BILES, S.P.; COTHREN, J.T. Flowering and yield response of cotton to application of mepiquat chloride and PGR-IV. Crop Science, v.41, p.1834-1837, 2001.

CARVALHO, M.C.S.; FERREIRA, G.B.; STAUT, L.A. Nutrição, calagem e adubação do algodoeiro. In: FREIRE, E.C. (Ed.). Algodão no cerrado do Brasil. Brasília: ABRAPA, 2007. p.581-647.

CATHEY, G.W.; MEREDITH JUNIOR, W.R. Cotton response to planting date and mepiquat chloride. Agronomy Journal, v.80, p.463-466, 1988.

COOK, D.R.; KENNEDY, C.W. Early flower bud loss and mepiquat chloride effects on cotton yield distribution. Crop Science, v.40, p.1678-1684, 2000.

GWATHMEY, C.O.; CRAIG JUNIOR, C.C. Managing earliness in cotton with mepiquat-type growth regulators. Crop Management, 2003. Disponível em: <http://www. plantmanagementnetwork.org/pub/cm/research/2003/ mepiquat/>. Acesso em: 10 dez. 2008. Doi:10.1094/ CM-2003-1222-01-RS.

HOAGLAND, D.R.; ARNON, D. The water culture methods for growing plants without soil. Berkeley: University of California, 1950. 32p.

LAMAS, F.M. Estudo comparativo entre cloreto de mepiquat e cloreto de chlormequat aplicados no algodoeiro. Pesquisa Agropecuária Brasileira, v.36, p.265-272, 2001.
LAMAS, F.M.; ATHAYDE, M.L.F.; BANZATTO, D.A. Reações do algodoeiro CNPA_ITA 90 ao cloreto de mepiquat. Pesquisa Agropecuária Brasileira, v.35, p.507-516, 2000.

MARUR, C.J. Fotossíntese e translocação de carboidratos em algodoeiro submetidos a déficit hídrico após a aplicação de cloreto de mepiquat. Revista Brasileira de Fisiologia Vegetal, v.10, p.59-64, 1998.

MCCARTY JUNIOR, J.C.; HEDIN, P.A. Effects of 1,1-dimethylpiperidinium chloride on the yields, agronomic traits and allelochemicals of cotton (Gossypium hirsutum L.), a nine year study. Journal of Agricultural and Food Chemistry, v.42, p.2302-2304, 1994.

NICHOLS, S.P.; SNIPES, C.E.; JONES, M.A. Evaluation of row spacing and mepiquat chloride in cotton. Journal of Cotton Science, v.7, p.148-155, 2003.

OOSTERHUIS, D.M. Growth and development of a cotton plant. Faytteville: University of Arkansas, 1992. 24p.

ORNELlAS, A.P.; HIROMOTO, D.M.; YUYAMA, M.M.; CAMARGO, T.V. de (Ed.). Algodão de Mato Grosso: qualidade e tecnologia ampliando mercados. Rondonópolis: Fundação MT, 2001. 237p. (Fundação MT. Boletim de pesquisa de algodão, 4).

PALLADINI, L.A. Metodologia para avaliação da deposição em pulverizações. 2000. 111p. Tese (Doutorado) - Universidade Estadual Paulista, Botucatu.

RAIJ, B. van; ANDRADE, J.C. de; CANTARELLA, H.; QUAGGIO, J.A. (Ed.). Análise química para avaliação da fertilidade de solos tropicais. Campinas: Instituto Agronômico, 2001. 285p.

REDDY, A.R.; REDDY, K.R.; HODGES, H.F. Mepiquat chloride (PIX)-induced changes in photosynthesis and growth of cotton. Plant Growth Regulation, v.20, p.179-183, 1996.

REDDY, V.R.; BAKER, D.N.; HODGES, H.F. Temperature and mepiquat chloride effects on cotton canopy architecture. Agronomy Journal, v.82, p.190-195, 1990.

SISTEMA brasileiro de classificação de solos. Brasília: Embrapa Produção de Informação; Rio de Janeiro: Embrapa Solos, 1999. 412 p.

SOARES, J.J. Fitorreguladores e remoção da gema apical no desenvolvimento do algodoeiro. Scientia Agricola, v.56, p.627-630, 1999.

SOUZA, F.S. de. Persistência de cloreto de mepiquat em plantas de algodão em função da precipitação. 2004. 59p. Dissertação (Mestrado) - Universidade Estadual Paulista, Botucatu.

SOUZA, F.S. de; ROSOLEM, C.A. Rainfall intensity and mepiquat chloride persistence in cotton. Scientia Agricola, v.64, p.125-130, 2007.

STEWART, A.M.; EDMISTEM, K.L.; WELLS, R.; YORK, A.C.; JORDAN, D.L. Wick applicator for applying mepiquat chloride on cotton: II. Use in existing mepiquat chloride management strategies. Journal of Cotton Science, v.5, p.15-21, 2001. 
TAIZ, L.; ZEIGER, E. Fisiologia vegetal. 3.ed. Porto Alegre: Artmed, 2004. 719p.

YORK, A.C. Cotton cultivar response to mepiquat chloride. Agronomy Journal, v.75, p.663-667, 1983.
ZANON, G.D. Manejo de cultivares de algodoeiro em densidade populacional variável com o uso de regulador de crescimento. 2002. 75p. Dissertação (Mestrado) - Escola Superior de Agricultura Luiz de Queiroz, Piracicaba.

Recebido em 19 de fevereiro de 2009 e aprovado em 29 de setembro de 2009 